\section{Hammerhead shark origins}

SIR - Knowledge of macroevolutionary sequences provides insight into the origin of biological innovation and the nature of selective forces responsible for the evolution of adaptations. Hammerhead sharks (Family Sphyrnidae) are characterized by the presence of a remarkable innovation: a laterally expanded head (termed the cephalofoil) in which there has been substantial reorientation of olfactory, optic and electric field sense organs. Because head shapes across contemporary species differ markedly in the extent of lateral elaboration, knowledge of phylogenetic relationships among species allows estimation of the trajectory of cephalofoil evolution.

Previous phylogenetic analysis of morphology ordered taxa according to the absolute degree of cephalofoil lateral expansion; namely, the species with the least laterally expanded head is

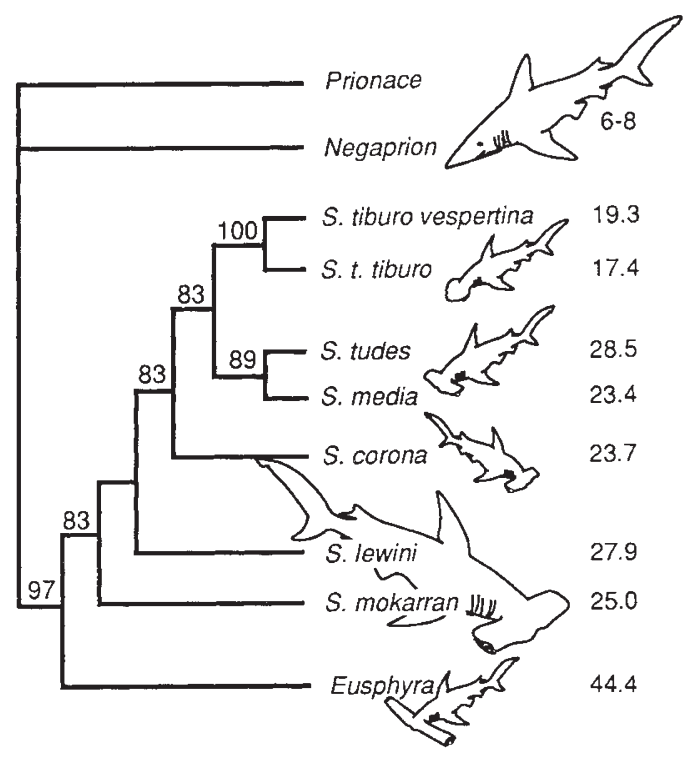

Phylogenetic hypothesis of relationships among species of Sphyrna and Eusphyra based on DNA sequence data. Phylogeny was inferred using the branch-andbound algorithm of PAUP (ref. 2) and using the neighbour-joining cluster algorithm on the matrix of corrected pairwise differences (implemented using PHYLIP; ref. 3). The neighbour-joining tree was identical to one of the two minimum-length topologies. This phylogenetic hypothesis is 35 steps shorter than the progressive evolution hypothesis ${ }^{1}$, a difference that is significant based on likelihood analysis ${ }^{3}$. Numbers along the branches are the percentage of times that that branch was found in 250 bootstrap replications. Only values greater than 50 are shown. Cartoons of sharks provide an indication of the relative size and head shape of the species. Average head widths (expressed as a percentage of body length) are given for each species (from ref. 4). Data are for sharks that are $60-70 \mathrm{~cm}$ in length. Protocols for sequencing shark DNA, the DNA data and additional details of methods of phylogenetic inference are available from the author on request. regarded as ancestral, the most derived lineage is characterized by the most exaggerated cephalofoil, and species with intermediately expanded heads are rdered so that increasingly derived taxa creasingly expanded heads ${ }^{1}$. This ypothesis is intuitively appealing because suggests that there has been directional election for lateral expansion ('improveent'), and that the variations present in emporary species are snapshots of ages in the progress of cephalofoil aboration. To test this hypothesis, base pairs from two itochondrial protein-coding genes for axa of hammerheads ( 7 species, $2 \mathrm{sub}$ pecies) across the full array of morpholPies and for two outgroups.

Phylogenetic analysis of the DNA data provided support for previous claims that hammerheads constitute a monophyletic group, implying a single origin of the cephalofoil (see figure). However, the ondrial DNA phylogeny suggests trikingly different sequence of hammerhead evolution than previously thought (see figure). Most notably, narrowheadedness (characteristic of Sphyrna tiburo) is derived, not ancestral. Furthermore, the lineage characterized by extreme head width (Eusphyra blochii) is most ancestral. These data provide no information about the origin of the cephalofoil, and they argue that the variation in extant taxa provides misleading information about the trajectory of morphological evolution either because of the tendency to align things in progressive series or the assumption of parsimony. Instead, the data suggest that once the cephalofoil had originated, head width (relative to body length) underwent exaggerated expansion (along the Eusphyra lineage), diminution (along the $S$. tiburo lineage), and remained unchanged at different times in hammerhead shark evolution.

The cephalofoil is thought to function as a bowplane to increase lift when the shark moves through the water and to enhance manoeuvrability when the shark twists, elevates or depresses its head. In addition, expansion of the head probably enhanced orientation and prey detection capabilities that would accompany increased number and/or separation of electric field, olfactory and ocular sense organs. Identification of at least two distinct functions provides the opportunity for selection to act in different directions, a possibility that may explain the existence of multiple cephalofoil designs. Descriptions of ontogenetic and morphological variation within and among species and observations of the comparative performance of sharks whose heads are of different shape should explain the presence of different cephalofoil designs.

\section{Andrew Martin}

Smithsonian Tropical Research Institute, Naos Marine Laboratory,

POBox 2072, Balboa,

Republic of Panama
1. Compagno, L. J.V. Sharks of the Order Carcharhiniformes (Princeton Univ. Press, 1988)
2. Swofford, D. Phylogenetic Analysis Using Parsimony (lllinois Natural History Survey, Champaign, Urbana. 1991)
3. Felsenstein, J. Phylogenetic Inference Package (University of Washington, Seattle, 1990)

4. Gilbert, C. R. Proc. US Natn. Mus. 119, 1-88 (1967).

\section{Estimating extinction rates}

SIR - Continuing patterns of human population growth and associated destruction of natural habitats lead many to believe that we are probably entering a period of mass extinction comparable in degree to, and swifter in time than, the great spasms of extinction in the geological past $\mathrm{t}^{1,2}$. The commonly cited estimates of extinction rates are, however, very rough; they derive mainly from species-area relations combined with projected rates of destruction of natural areas $^{2,3}$. Here, we use data compiled by the World Conservation Monitoring Centre (WCMC) to make some different, but still very rough, assessments of impending extinction rates.

Specifically, these data include documented animal and plant extinctions since 1600 (ref. 4); 'Red Lists' of animal species threatened with extinction, compiled every two years by the WCMC using the International Union for the Conservation of Nature's (IUCN) categories 'probably extinct', 'endangered' (survival unlikely if causal factors continue) and 'vulnerable' (likely to become 'endangered' if current trends continue), along with 'rare' (but not necessarily threatened), 'status unknown' and 'not threatened' (refs 5-7); and the WCMC database on seed-bearing plant species threatened with extinction, which uses comparable categories of threat.

Table 1 summarizes these data, showing the proportions of major plant and animal taxa that have become extinct since 1600 or are currently threatened. Although crude, these figures are illuminating, even though they mainly show the enormous variations in the attention different taxa have received $^{8}$. Even for comparatively well-studied groups like birds and mammals, the numbers are thought to be serious underestimates because many tropical species have not received the 